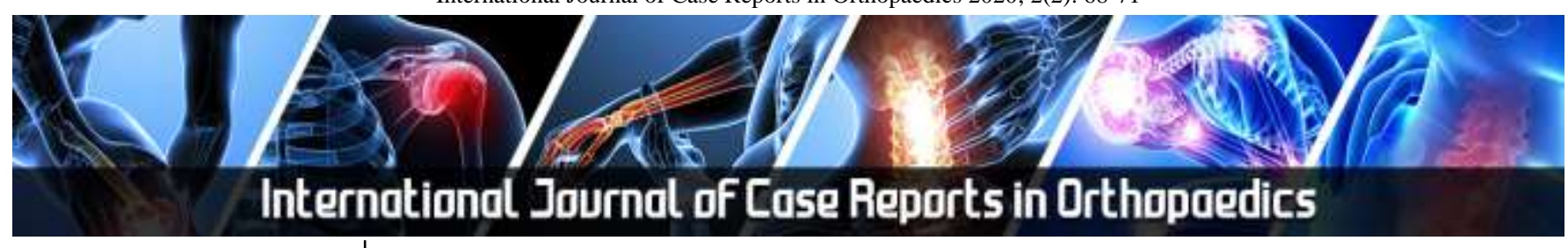

E-ISSN: 2707-8353 P-ISSN: 2707-8345 IJCRO 2020; 2(2): 68-71 Received: 09-05-2020 Accepted: 12-06-2020

Francisco de Assis Serra Baima Filho

Orthopedist and

Traumatologist with

Subspecialty in Orthopedic

Oncology, Federal University

of Maranhão (UFMA), Brazil

Joel Pires Ribeiro

Currently studying Medicine at the Federal University of Maranhão (UFMA), Brazil
Corresponding Author: Francisco de Assis Serra Baima Filho

Orthopedist and

Traumatologist with

Subspecialty in Orthopedic

Oncology, Federal University

of Maranhão (UFMA), Brazil

\section{Tuberculosis osteomyelitis in foot and ankle bones: A case report}

\author{
Francisco de Assis Serra Baima Filho and Joel Pires Ribeiro
}

DOI: https://doi.org/10.22271/27078345.2020.v2.i2b.30

\begin{abstract}
Osteomyelitis due to tuberculosis in foot and ankle bones is an uncommon pathology (less than 1\%) and in most patients the symptoms are non-specific, requiring a high index of suspicion for the diagnosis. It is most common in children or in people between thirty and forty years old. Bone biopsy is the gold standard for diagnosis. The recommended treatment is medication with surgery indicated in the sequelae. Late diagnosis and inadequate treatment can lead to significant morbidity and deformities. This research aims to report the case of a 31-year-old patient with bone tuberculosis, reporting diagnosis, treatment and evolution, in addition to presenting a literature review.
\end{abstract}

Keywords: Bone tuberculosis (MeSH: D014394); rare disease (MeSH: D035583); case study (MeSH: D002363)

\section{Introduction}

According to the World Health Organization (WHO), annually, there are approximately 6 million new cases of tuberculosis (TB) worldwide. One third of the entire infectious TB load of the American continent circulates in Brazil ${ }^{[1]}$.

Among all TB cases, about $10 \%$ correspond to extra pulmonary origin. Skeletal impairment represents about $3 \%$ of this pathology and foot and ankle bone involvement affects only $0.1 \%$ to $0.3 \%$ of all patients with extra pulmonary form ${ }^{[2]}$. The calcaneus region is the most commonly involved, followed by mid-tarsus, lisfranc and ankle joints ${ }^{[3]}$. The disease may have multifocal foot involvement ${ }^{[4]}$.

The objective of the article is to report a rare case of a patient who had osteomyelitis due to tuberculosis in the foot and ankle region, presenting clinical characteristics, diagnostic tests, treatment and complications of this condition, in addition to presenting a literature review.

\section{Case study}

CBOS, male, 31 years old, had a history of pain and swelling in his right ankle and foot with onset of symptoms in February 2017 which worsened over time. There was no history of trauma, weight loss or night sweats. He also reported receiving some medication (such as anti-inflammatories and painkillers) prescribed by different doctors, however, without effect. After seven months of evolution, the foot began to discharge a secretion.

Upon physical examination (performed in September 2017), a tumor was found in patient's anterior ankle and medial region of the calcaneus, with an active fistula and outflow of blood-purulent secretion in the latter region. In addition to the difficulty of sustaining load and pain when moving the tibiotalar and subtalar joints.

Laboratory tests did not show any changes, even HIV serology was negative.

The MRI exam showed nonspecific signs (Figures 1).

Thus, the patient underwent an incisional tumor biopsy, the diagnosis of which was confirmed by a histopathology exam for tuberculosis. Soon, he was submitted to drug treatment with the rifampicin, pyrazinamide and isoniazid regimen, for a period of 9 months. The patient evolved well with the treatment, as he eradicated the infection and regressed the tumor's volume (the foot has characteristics similar to the contralateral foot) and decreased pain intensity and frequency. However, arthrosis and bone deformity formation (Figures 2 and 3) was an after-affect along with complaints of pain when taking long walks and standing in a standing position for a long time. 


\section{Results and Discussions}

For the research, quantitative and exploratory methodologies were used with the collection of medical records and bibliographic research. The research platform used was PUBMED, selected articles that only commented only TB osteomyelitis in foot and ankle bones, and in the English language. In total there are six case reports and three are literature reviews from 2013 to 2018.

TB is a disease caused by Mycobacterium tuberculosis that can affect a number of organs and/or systems ${ }^{[1]}$. The osteoarticular form is the fourth most prevalent location, after pulmonary, urogenital and lymphatic locations ${ }^{[5]}$. It is most common in children or in people between thirty and forty years old, no gender predominance ${ }^{[1]}$. The patient in this research was in the predominant age group for this pathology. TB osteomyelitis in the foot and ankle region is usually detected at a more advanced stage due to sluggish evolution and nonspecific signs and symptoms common to other pathologies such as pyoarthritis, actinomycosis, mycetoma, rheumatoid foot, giant cell tumor, chondroblastoma, among others ${ }^{[3,6,7]}$. The most common symptoms include pain and edema lasting from 1 to 120 months ${ }^{[8]}$. Some patients have sinus drainage and/or a tumor in the form of a "cold abscess" [2]. Other characteristics that should raise suspicions: HIV-positive status, recent immigration or prolonged trips to a country of high prevalence and exposure to possibly positive individuals ${ }^{[9]}$. In the reported case, the patient took seven months for the diagnosis, had nonspecific signs such as edema and pain in addition to "cold abscess" formation and a negative result for HIV.
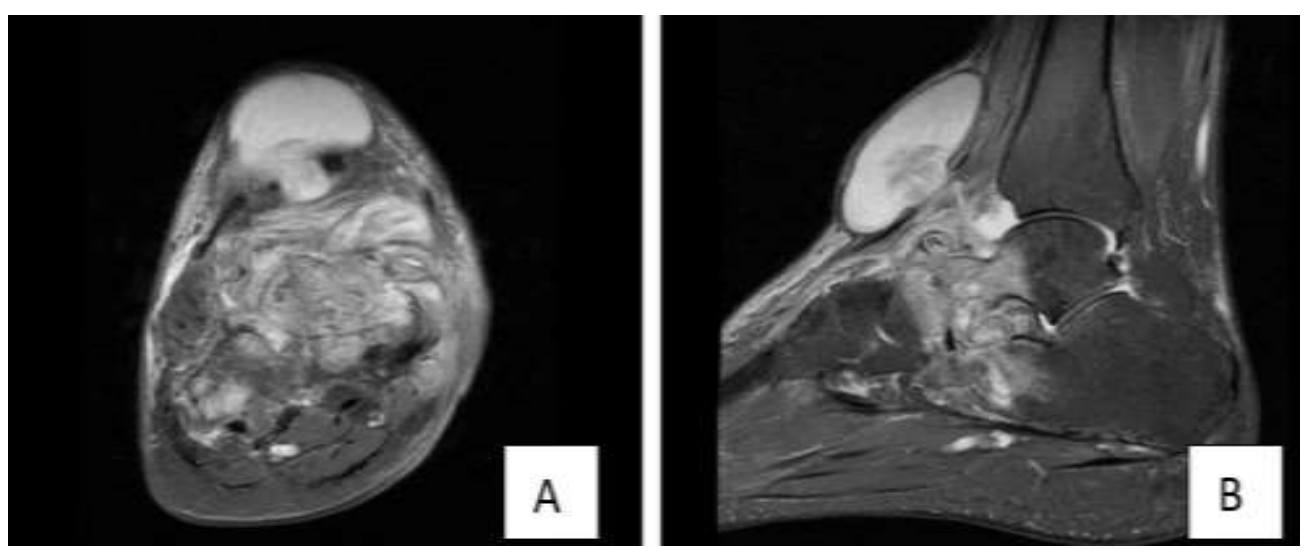

Fig 1: MRI of the right ankle before drug treatment. A: axial cut at T2 weighting. B: sagittal cut in T2 weighting. An extensive collection is observed in the subcutaneous region of the dorsal region of the right ankle, with a fistulous path with the talonavicular joint, highlighting the presence of synovitis and joint effusion in the calcaneocuboid and talonavicular joints and between the cuboid and the bases of the fourth and fifth metatarsals. The presence of subchondral bone erosions in the metatarsals, cuboid, navicular and talus also stands out

Table 1 shows the main findings in the literature in relation to laboratory, image and histopathological examination.

The recommended standard treatment is medication for 6 months of isoniazid and rifampicin, including supplementation with pyrazinamide and ethambutol in the first 2 months ${ }^{[9]}$. The scheme can continue for up to 24 months in cases of inadequate and/or incomplete treatment that resulted in the development of resistant bacterium ${ }^{[2]}$. Surgical treatment is reserved for cases that evolved with sequelae such as arthrosis and bone deformities ${ }^{[2]}$. The reported patient had eradicated the infection with drug treatment for a period of nine months with isoniazid, rifampicin and pyrazinamide.

The rarity of the problem and low index of suspicion lead to delays in diagnosis and are generally associated with the development of exuberant bone lesions, which involve local cartilage and lead to structural and functional joint degeneration ${ }^{[9]}$. The patient in this report evolved with joint sequelae of the foot and ankle such as bone deformities and areas of arthrosis. The patient in this case report had a history similar to the cases described in the literature such as the clinical examination with nonspecific signs, poorly clarified imaging tests and late diagnosis being confirmed by histopathological examination. Today, he complains of pain when walking long distances and staying in the orthostatic position for a long time and on imaging exams with signs of arthrosis and bone deformities. He is undergoing drug treatment with analgesics, restriction in walking long distances and, at the moment, without surgical indication for arthrosis treatment. 


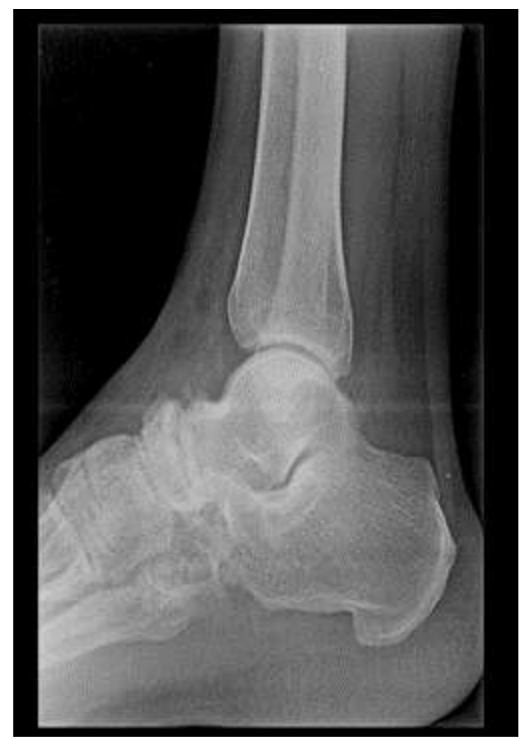

Fig 2: X-rays of the right foot in profile performed after drug treatment where the presence of a deformity with navicular flattening associated with sclerosis and subchondral erosions in the talonavicular, calcaneocuboid joint and between the cuboid and metatarsals is observed
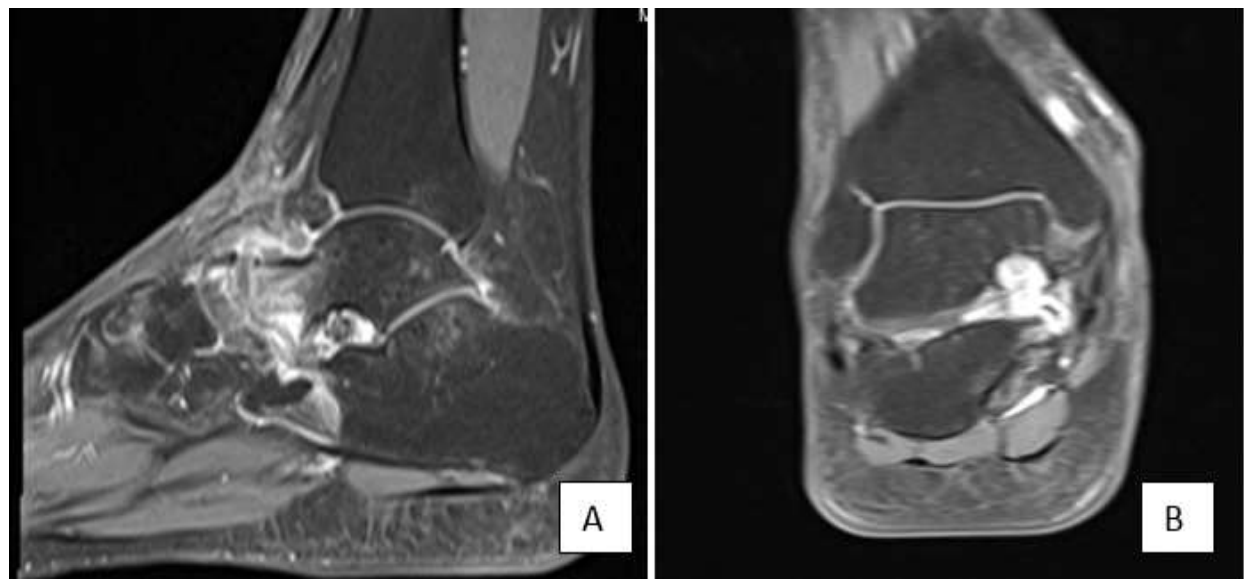

Fig 3: MRI of the right ankle in sagittal section (Figure A) and in coronal section (Figure B), both in T2 weighting, performed after drug treatment in which they show exuberant synovial thickening in the talocalcananeavicular, calcaneocuboid and cuboid joints at the 4th and 5th metatarsal bases, and extensive osteochondral erosions and tarsal canal synovitis.

Table 1: It lists the main findings found in the literature review of each exam in the evaluation of a patient with osteomyelitis due to TB in the foot and ankle bones

\begin{tabular}{|c|c|}
\hline Exams & Main findings \\
\hline Laboratory & Inflammatory markers (ESR and CRP) are not specific ${ }^{[2]}$. \\
\hline Radiography & Demonstrates diffuse osteoporosis, areas of bone destruction with or without bone sequestrum ${ }^{[3]} \cdot$ \\
\hline Tomography & It provides an idea of the extent of bone damage, especially in cases where MRI is not available ${ }^{[6]}$. \\
\hline Resonance & $\begin{array}{c}\text { Cortical reaction and intense bone marrow edema. In addition to demonstrating the spread in soft tissues and outlining } \\
\text { the extent of the disease }{ }^{[2,10]} .\end{array}$ \\
\hline Histology & Granulomatous cells with epithelioid macrophages and giant Langhans cells around caseous necrosis $^{[7]}$. \\
\hline
\end{tabular}

\section{Conclusion}

Foot and ankle TB osteomyelitis is a rare condition with clinical signs and nonspecific imaging tests, which leads to delayed diagnosis. This delay can lead to sequelae such as joint deformities and chronic pain. Thus, the research emphasizes the importance of the histopathological exam to be performed as soon as possible in order to avoid or minimize the sequelae in patients with symptoms similar to the one described.

\section{References}

1. Ministério da Saúde. Manual de recomendações para o controle da tuberculose no Brasil [Internet]. Ministério da Saúde 2019, 364. Available at: https://www.telelab.aids.gov.br/index.php/bibliotecatelelab/item/download/172_d411f15deeb01f23d9a5566 19ae965c9

2. Wicks L, Faroug R, Richler-Potts D, Bowden A, Issac R, Mangwani J. Diagnosis and treatment of tuberculosis of the foot and ankle-A literature review. Foot [Internet] 2018;37(March):105-12. Available at: https://doi.org/10.1016/j.foot.2018.07.005

3. Upadhaya GK, Jain VK, Sinha S, Naik AK. Isolated calcaneocuboid joint tuberculosis: A rare case report. Foot [Internet] 2013;23(4):169-71. Available at: http://dx.doi.org/10.1016/j.foot.2013.10.001 
4. Dhillon MS, Agashe V, Patil SD. Role of Surgery in Management of Osteo-Articular Tuberculosis of the Foot and Ankle. Open Orthop J 2017;11(1):633-50.

5. Lemnouer A, Frikh M, Belfquih B, Jaafar A, Bouya A, Jidal $\mathrm{M}$ et al. Navicular tuberculosis: $\mathrm{A}$ rare localization of bone tuberculosis. ID Cases [Internet] 2015;2(3):80-2.

Available

at: http://dx.doi.org/10.1016/j.idcr.2015.08.002

6. Vijay V, Gupta N, Vaishya R. Tuberculosis around the tarsal navicular: A rare entity. Foot [Internet] 2016;28(1210):20-5. Available at: http://dx.doi.org/10.1016/j.foot.2016.09.003

7. Gursu S, Yildirim T, Ucpinar H, Sofu H, Camurcu Y, Sahin $\mathrm{V}$ et al. Long-term follow-up results of foot and ankle tuberculosis in Turkey. J Foot Ankle Surg 2014;53(5):557-61.

8. Woo JC, Seung HH, Jong HJ, Bom SK, Jin WL. Diagnostic dilemma of tuberculosis in the foot and ankle. Foot Ankle Int 2008;29(7):711-5.

9. Korim M, Patel R, Allen P, Mangwani J. Foot and ankle tuberculosis: Case series and literature review. Foot [Internet] 2014;24(4):176-9. Available at: http://dx.doi.org/10.1016/j.foot.2014.07.006

10. Brew CJ, Rao V, Shanker J. Tuberculosis infection of the talonavicular joint. Foot [Internet] 2010;20(4):1468.

Available

at: http://dx.doi.org/10.1016/j.foot.2010.08.001 